\title{
A Study on Smart Helmet to Efficiently Cope with the Operation and Safety of Workers in Industrial Settings
}

\author{
Junghwan Byeon ${ }^{1}$, Mun-Seok Jang ${ }^{2}$, Sang-Won Choi ${ }^{3}$, Hyun Dong $\mathrm{Yoo}^{4}$ and \\ Eung-Hyuk Lee ${ }^{5 *}$ \\ ${ }^{5}$ Dept. of Electronic Engineering, Korea Polytechnic Univ. Siheung, Korea \\ ${ }^{2}$ Dept. Of Electronic Engineering, Dongeui Institute of Technology, Pusan, Korea \\ ${ }_{1,3,4}$ Korea Occupational Safety and Health Agency, Korea \\ ${ }^{1}$ bjh6918@kosha.or.kr, ${ }^{2}$ msjang@dit.ac.kr, ${ }^{3}$ swchoi@kosha.or.kr, \\ 4yoohd@kosha.or.kr, ${ }^{5}$ ehlee@kpu.ac.kr
}

\begin{abstract}
Among victims of occupational accidents in South Korea for the past seven recent years (2008-2014), victims of accidents in the construction industry accounted for $27.35 \%$ and deaths among them accounted for $43.75 \%$, representing the largest proportion of victims. Deaths by crashing accounted for the largest proportion of deaths, and the most frequently injured part of the body was the head. This study proposed and developed a smart helmet, which is an industrial safety helmet with a detachable smart module that uses a variety of sensors to collect workers' working status and, situations relating to safety for workers' safety in industrial sites. The proposed safety helmet is entitled to protective gear safety certification in South Korea, and the replacement cycle for the safety helmet is approximately three months, while there is no expiration term for the wireless module within the helmet. The use of the proposed safety helmet would allow the injured to receive emergency aid within the golden time when an accident takes place. Moreover, the helmet minimizes the loss of life on a national level, the production costs and the production time with the helmet may be reduced within the conventional industrial settings, and the development of the helmet itself may contribute to the advancement of smart technology.
\end{abstract}

Keywords: Walking assist, Fall, Laser Range Finder, Stability

\section{Introduction}

Recent industrial settings are undergoing great changes through growth in scale, use of machinery, and diversification, and the industry is seeking ways to reduce construction costs and shorten construction periods following the deterioration of profitability. Due to these reasons, accident risks in industrial settings are increasing unprecedentedly while occupational accidents are taking places continuously. While the main reasons of the occupational accidents may be simplified into two categories: reduction of cost and time the actual causes of occupational accidents are complex and combination of multiple factors. The occupational accidents that took place in South Korea for recent seven years(2008-2014) indicate that victims of accidents in the construction industry accounted for $27.35 \%$, and deaths among them accounted for $43.75 \%$, representing the largest proportion of victims. This is attributed to potential risks from the construction industry due to increases of building heights, use of machinery, increases of the complexity of work, lack of capacity to control safety against the increase of higher working operations, diversity of hoisting equipment, and the application of new construction methods.

Received (December 18, 2017), Review Result (February 23, 2018), Accepted (March 2, 2018) 
Under the type of recent accidents that led to death in the construction industry, deaths by crashes have accounted for the highest rate at 54.53\% [1]. The safety helmet is a device that protects the worker's head against objects flying into the head or falling from above, or when the worker falls. It also protects the worker against electric shock. Furthermore, the smart helmet refers to the safety helmet for industrial use equipped with various sensors to monitor a worker's operational status and situational information in relation to safety followed by the recent advancement of wireless communication technology.

To reduce accidents in industrial settings, it is important to provide safety knowledge and techniques to workers through various safety training programs in the field. However, it is also important to have a system to operate rescue activities within the golden time by checking the emergency situation of the worker whenever an accident occurs.

This study proposes a system obligating workers in industrial settings to wear the smart helmet, which has various built-in sensors to tell the supervisor in the safety office of the safety status of the worker through wireless communication. The proposed system adds an image sensor, GPS module, illumination sensor, LED lamp, and emergency button to the safety helmet to form a smart safety helmet. The system transmits the worker's operating environment and emergency state data to the safety supervisor in the safety office through the Zigbee wireless network communication following the IEEE 802.15.4 communication protocol, and so that the supervisor can check the location of the GPS module and the router in the wireless network to estimate the position of the worker and deploy rescue operations within the golden time when an emergency situation occurs.

This study is composed of the following parts. Chapter 2 introduce the smart helmet in the smart safety control system, Chapter 3 explain how the smart helmet is composed and how it operates within a scenario, and Chapter 4 discusses the smart helmet as developed. Finally, Chapter 5 offers a conclusion.

\section{Smart Helmets in the Smart Safety Control System}

\subsection{Related Studies}

Many studies have been conducted to reduce accidents. Yeong-soo Kim divided safety control into five steps by proposing a safety control guide model and developed a system for managing safety information [2] and Seong-seok Goh developed a safety information system that provides safety information combined with examples of accidents [3]. Hong-seop Ahn divided safety control into four stages to enhance the effectiveness and efficiency of safety control activities [4]. Bo-hyeok Ryu examined the actual conditions when using safety helmets during high place operations through a survey [5], Jae-hee Jeong examined the actual conditions of manufacturers, distributors, and users of protective gear and devices, including safety helmets, and proposed a plan to promote the market [6]. Jin-hyeon Kim suggested the certification criteria (plan) for light safety helmets and others as a part of light protective gear for the service industry, current safety helmet related laws, and the revision (plan) of criteria [7]. Don-heung Choi examined the use of personal protective gear such as safety helmets regarding workers operating on the roof and suggested a safety model for preventing crash accidents during rooftop operations [8]. However, these studies are related with safety control activities that are incapable of knowing the circumstances of the worker at the actual construction site; they can only cope with accidents in their existing format.

A number of studies have been conducted to combine smart technology with safety helmets for industrial use. Sang-won Choi suggested the development of 
safety technology and a safety policy through a study of the actual conditions for the development of safety technology using IT [9], and In-yong Song and Moon-seok Jang developed a device that checks whether the worker is wearing a safety helmet and a system which monitors workers who are not wearing chinstraps and sends data to the central control PC $[10,11]$. Seok-yong Hong developed a wireless communication module using the $400 \mathrm{MHz}$ band frequency for a device that checks whether the safety helmet is being worn [12] and proposed evaluation criteria for the reliability of the smart module applied to safety helmets for industrial use. However, these studies are limited to systems that check the safety helmet's chinstrap and are unable to check the working environment or safety related situations.

Therefore, this study proposes a smart helmet that can monitor and control workers' activities for safety and cope with the working environment and safety related situations.

\subsection{Smart Safety Control System}

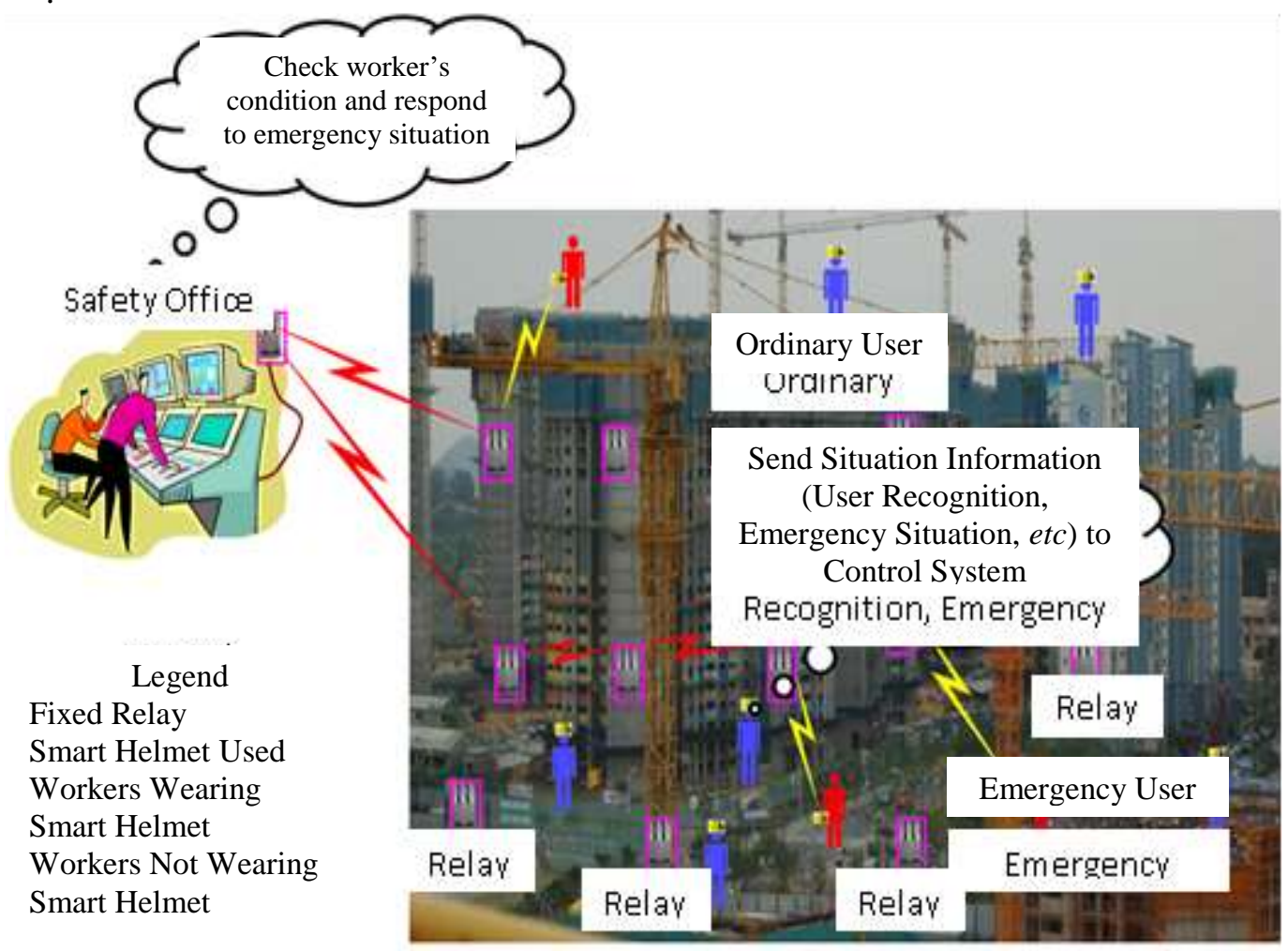

Figure 2.1. Organization of the Smart Safety Control System

Figure 2.1 represents the Smart Safety Control System. This system comprises a smart helmet, wireless communication relay, wireless communication gateway, and a safety control program. The smart helmet for industrial use sends various data, including the worker's situational information (movement, whether the worker is wearing a chinstrap, etc.) and spot information (image, brightness, altitude) to the safety control program of the safety manager using a wireless sensor network. The wireless communication relay connects the smart helmet for industrial use with a wireless communication gateway and expands the communication range between the safety office and the user's operating environment. Lastly, the safety control program provides a user interface to analyze the data collected from the smart helmet for industrial use and provides data to the safety control supervisor. 


\subsection{Smart Helmet Requirements}

In the smart safety control system, it is essential for workers to wear the smart helmet to obtain situational information on safety and detect accidents. In order to apply the smart helmet as proposed in this study in actual industrial settings, the sensor and communication modules must be produced in a structure that can be mounted to or dismounted from the safety helmet for the following reasons. First, the most commonly used safety helmet in South Korea is the ABE that can endure a $7,000 \mathrm{~V}$ or higher voltage. According to South Korea's Notification on Mandatory Protective Gear Safety Certification (Ministry of Employment and Labor Notification No. 2012-83), the general structure of the AE type safety helmet shall not use metal parts. As the ABE type helmet also needs to meet conditions for the AE type, ABE safety helmets shall not use electronic parts. However, this condition is not applied to detachable products, and electronic parts may be used on detachable parts.

Second, the price of the safety helmet produced in China is less than 10,000 KRW, while the helmet produced in South Korea costs more than 10,000 KRW, with the most expensive sold at 20,000 - 30,000 KRW. The price of safety helmets circulating in South Korea is $10,000 \mathrm{KRW}$ or lower on average. While workers sometimes purchase their own safety helmets outside Korea, business owners also purchase and distribute safety helmets to workers. Business owners tend to purchase safety helmets at the lowest possible price. Therefore, the smart helmet should be distributed at the lowest possible price to promote distribution.

Third, the replacement cycle for the safety helmet is very short three to six months, due to the staining of the inner surface as well as the whole helmet and bacteria issues. No one would want to purchase a safety helmet equipped with various sensors, controllers, and communication modules to be replaced within every six months. Therefore, the smart helmet must be developed as an existing safety helmet with a detachable smart module so that they can replace the safety helmet following an ordinary replacement cycle and reuse the smart module with the replaced safety helmet to enable semi-permanent use.

Finally, it is preferable for all workers to be given a smart helmet depending on their working environment and habits. However, those who purchase smart helmets wish to lessen the burden of purchasing smart helmets and reduce accident rates by distributing it only to workers operating in areas where accidents have happened frequently and among workers who have a habit of not wearing a safety helmet, rather than to all workers. Therefore, it is necessary to eliminate the difference in design between existing safety helmets and smart helmets.

Since including all sensors to the smart module would increase the production cost and selling price, it is deemed appropriate to build smart modules for each function to provide only the essential functions required by customers.

Various sensors and communication modules that constitute the smart helmet to recognize workers' situation and safety are as follows:

- Inertia Measurement Unit (IMU) - Measures the user's movement to determine emergency status

- Illumination Sensor - Measures the ambient brightness to turn on the high brightness LED of the safety helmet when it is dark to check the user's position

- Barometric Sensor - Checks the worker's operating position (height) or determines where the worker is located (floor number) during an emergency

- Temperature Sensor - Measures the ambient temperature of the worker

- Image Sensor - Sends images recorded under emergency situations or from working environments in image cuts 
- Reed Sensor - Checks whether the worker is wearing the chinstrap of the safety helmet

- $\quad$ EMG Switch - The worker can press this switch under emergency situations to notify an emergency state

- High Brightness LED - Brightens areas when it is dark or enables for a fast search of the worker in an emergency situation

- Communication Module - Sends data collected from various sensors to the safety office

\section{Organization and Operation Scenario of the Smart Helmet}

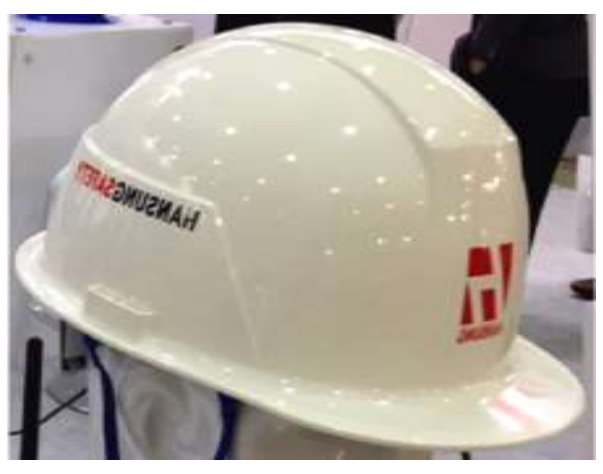

(a) Safety Helmet

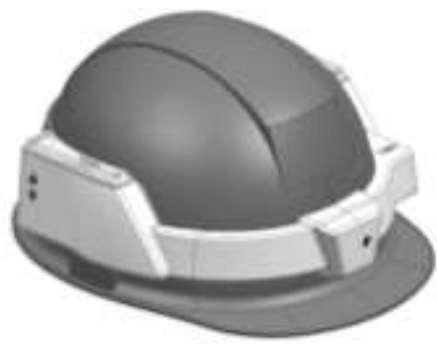

(b) Smart Helmet

Figure 3.1. Smart Helmet

\subsection{Organization of the Safety Helmet}

The safety helmet of the smart helmet must be an ordinary commercial safety helmet as in Figure 3.1(a) with a structure appropriate for mounting the smart module onto it, and must satisfy the standards for mandatory protective gear safety certification. Furthermore, the helmet must maintain the center of gravity and shall not be tilted to one side when the smart module is mounted as in Figure 3.1(b). It is important that the safety helmet has small slots on both sides around the ear area to connect to and disconnect from the smart module as necessary.

\subsection{Organization of the Smart Module}

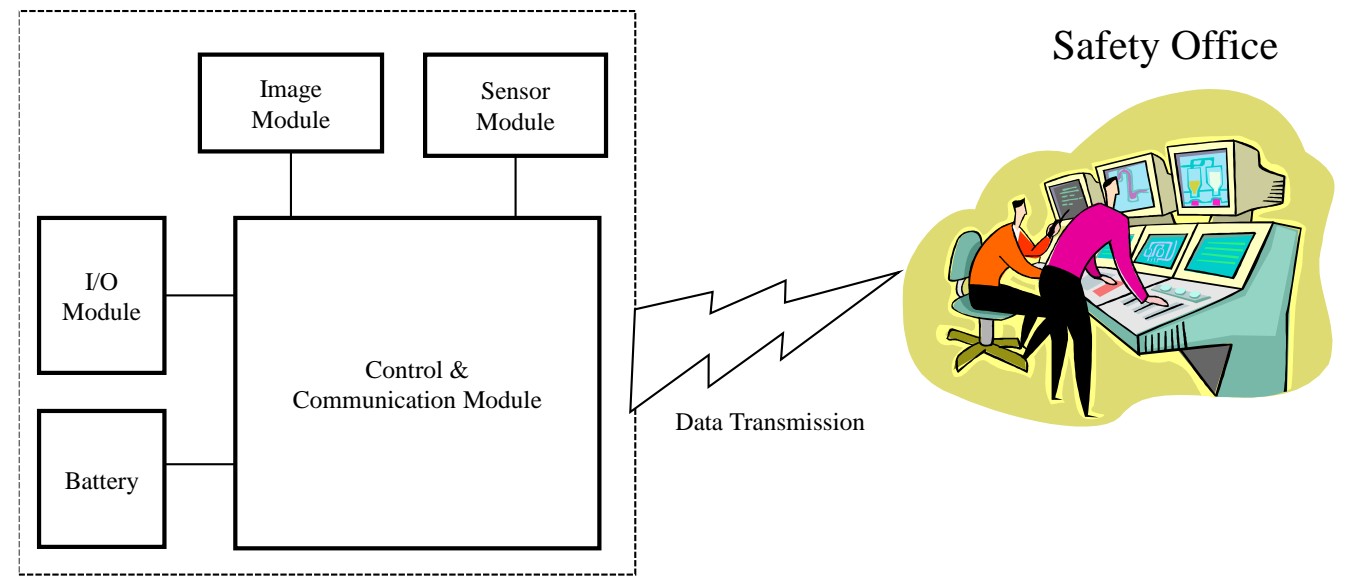

Figure 3.2. Organization of the Smart Module 
Figure 3.2 represents each functional module of the smart module. Battery supplies power to the entire smart module. The I/O module is connected to the external terminal in the smart helmet. This is composed of a battery charging terminal, power switch, and the reed sensor, which determines whether the user is wearing the chinstrap. The image module is used to send images recorded from the environment surrounding the user and during emergency situations. The sensor module is composed of the sensors built into the smart module, such as the inertia measurement unit (IMU), illumination sensor, barometric sensor, and temperature sensor. The sensor module can be composed of various sensors required for various operations and environments. The control \& communication module sends the data collected from the $\mathrm{I} / \mathrm{O}$ module, image module, and sensor module to the safety office using a wireless communication network.

\subsection{Operation Scenario of the Smart Helmet}

The operation of the smart module attached to the smart helmet is divided into initial operation, event operation, and control \& communication operation. Figure 3.3 represents the internal operation process of the smart helmet. The initial operation is to check the operation of all parts built into the smart module when the worker wears the smart module and to check whether communication is established. The event operation is designed to check the situation in the surrounding environment or recognize emergency situations when they occur. Major events include the pressing of the emergency button, the pressing of the image transmission button, a loose chinstrap, ambient brightness measurement, altitude measurement, and ambient temperature measurement. Once the emergency button is pressed, emergency situation data are sent to the safety office immediately. When the image transmission button is pressed the image is captured through the image sensor attached to the smart module and sent to the safety office. Any loose chinstrap is automatically reported to the safety office when the worker arbitrarily loosens the chinstrap during work hours, and the module also automatically measures the ambient brightness and altitude at regular intervals (periods) and send the data to the safety office. Such information can be collected from the safety office by requesting the helmet for information of the worker's surroundings. Lastly, the control \& communication operation collects and analyzes the data of various sensors collected from events and sends them to the safety office through a wireless communication network.

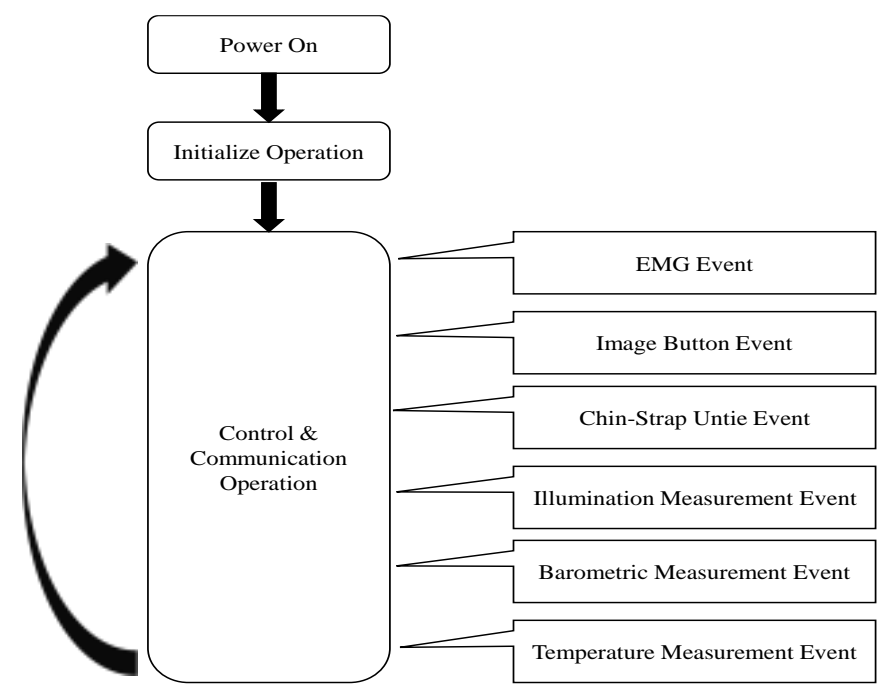

Figure 3.3. Internal Operation Process of the Smart Helmet 


\section{Implementation of the Smart Helmet}

\subsection{Design of the Smart Module for the Smart Helmet}

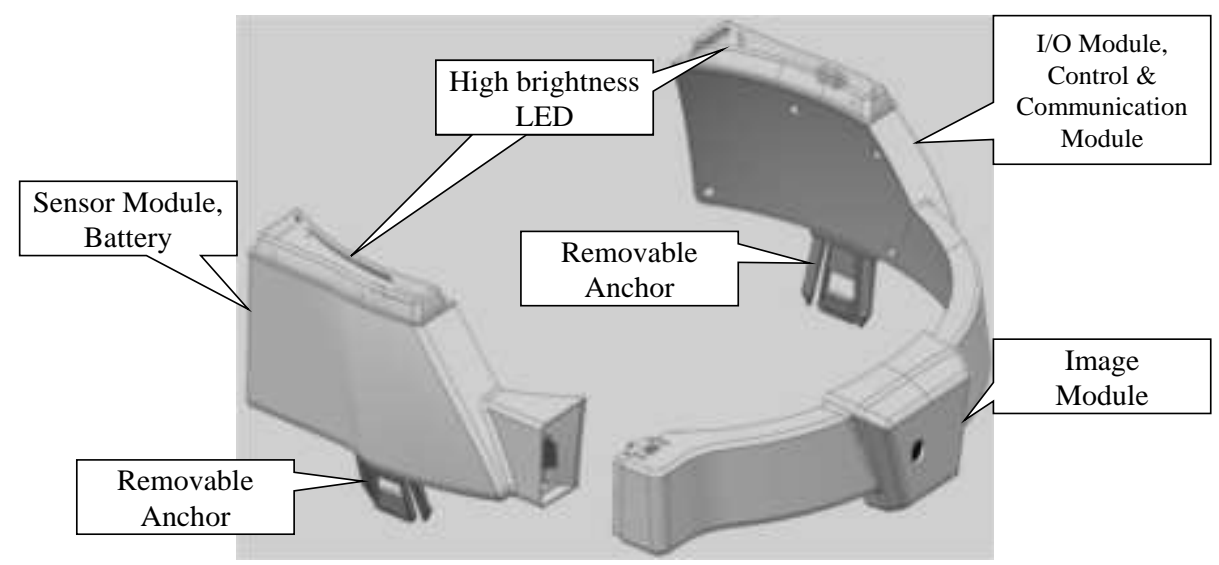

Figure 4.1. Assembly of a Functional Module in the Smart Module

Table 4.1. Specifications of the Functional Module in the Smart Module

\begin{tabular}{|c|c|}
\hline $\begin{array}{l}\text { Name of } \\
\text { Functional } \\
\text { Module }\end{array}$ & Specification \\
\hline Battery & Lithium Ion Battery, $560 \mathrm{~mA} \times 2=1120 \mathrm{mAh}$ \\
\hline I/O Module & $\begin{array}{ll}\text { - } & \text { Battery Charging Circuit: Microchip MCP73831 } \\
\text { - } & \text { Battery Charging Terminal } \\
\text { - } & \text { Reed Sensor I/O Terminal } \\
\text { - } & \text { High Brightness LED: Red (Power Display), Green (High } \\
\text { Brightness Display) }\end{array}$ \\
\hline Sensor Module & $\begin{array}{l}\text { - Inertia Measurement Unit: MCU-9250 (Triaxial } \\
\text { Gyroscope, Triaxial Acceleration, Triaxial Magnetometry) } \\
\text { B Barometric Sensor: SCP100 (Measures pressure for the } \\
\text { altitude for each centimeter by measuring } 30-120 \mathrm{KPa} \text { of } \\
\text { atmospheric pressure. Includes a temperature sensor) } \\
\text { - Temperature Sensor: Uses SCP100 }\end{array}$ \\
\hline Image Module & $\begin{array}{l}\text { Uses a VC0706 Digital Video Processor. Captures and } \\
\text { transmits QVGA (320x240) image (115,200bps) }\end{array}$ \\
\hline $\begin{array}{l}\text { Control \& } \\
\text { Communication } \\
\text { Module }\end{array}$ & $\begin{array}{l}\text { - Main Controller: STM32L152 (Cortex-M3) } \\
\text { - Wireless Communication: CC2531 (Supports Zigbee Pro } \\
\text { Network of the IEEE 802.15.4 Communication Protocol) } \\
\text { - Wireless Communication Antenna: Uses FPCB+U.FL } \\
\text { Coaxial Cable }\end{array}$ \\
\hline
\end{tabular}

For the smart module in the smart helmet, the layout of the functional module explained in Chapter 3.2 is important. In this study, the functional module was placed in the smart module as shown in Figure 4.1. The smart module is composed of three blocks. The left block is composed of a sensor module and battery, the center block is composed of the image module, and the right block is composed of 
an I/O module and control \& communication module. High brightness LED is placed above the left and right blocks, and the removable anchors are placed under the left and right blocks to mount to and dismount from the safety helmet.

Table 4.1 represents the specifications of each module of the smart module. The battery here can be used continuously when it is assumed that the worker operates eight hours a day, on average. The inertia measurement unit of the sensor module assumes an emergency situation if the user does not move for at least 5 minutes. The barometric sensor is designed to check the floor number in the operating area in high rises, such as on a construction site, using Formula (4.1).

$15^{\text {th }}$ Floor or Lower

Current Floor $(\mathrm{fn})=$ Current Altitude $(\mathrm{m}) / 0.26$

$16^{\text {th }}$ Floor or Higher

Current Floor $(\mathrm{fn})=($ Current Altitude $(\mathrm{m})-(0.26 \times 15)) / 0.28$

The illumination sensor measures the brightness of the working environment and determines whether the environment is appropriate for work. According to occupational safety and health standards, the brightness of the working environment shall be maintained at 150lux for electric machinery and equipment operation, 75lux for harbor loading and unloading, 750lux for super precision work, 150lux for ordinary environments, and 75lux or higher for other operations. The temperature sensor is designed to determine whether the environment is appropriate for work by measuring the temperature in the working environment. The temperature of the working environment shall be maintained at $37^{\circ} \mathrm{C}$ or lower. When the temperature of the working environment fluctuates drastically by $1^{\circ} \mathrm{C}$ or greater in 10 seconds, the situation is treated as an emergency and related data are sent to the safety office.

\subsection{Implementation of the Smart Module for the Smart Helmet}

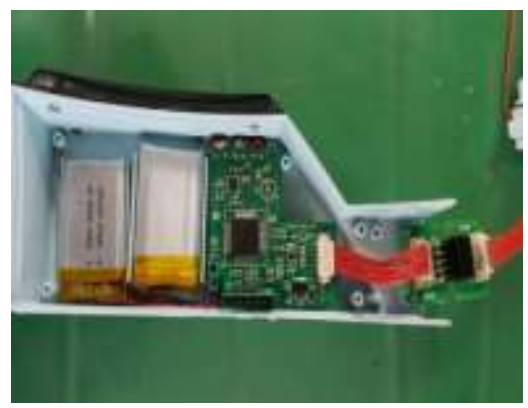

(a) Left Block

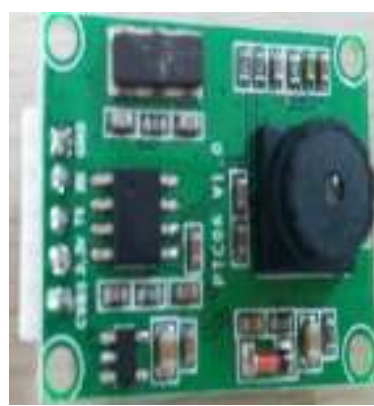

(b) Center Block

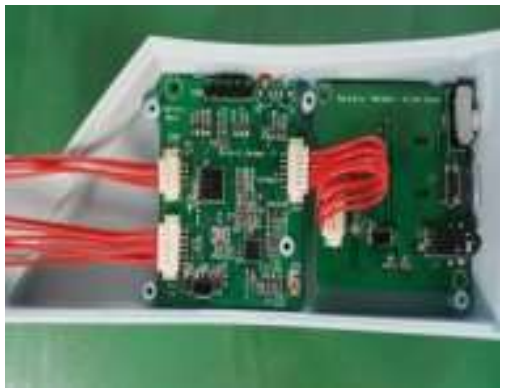

(c) Right Block

Figure 4.2. Implementation of the Smart Module

Figure 4.2 represents the smart module developed for the smart helmet. Figure 4.2(a) shows the left block composed of a battery and sensor module, Figure 4.2(b) shows the center block composed of an image module, Figure 4.2(c) shows the right block composed of an I/O module, control \& communication module, and Figure 4.3 is the fully assembled smart helmet. 


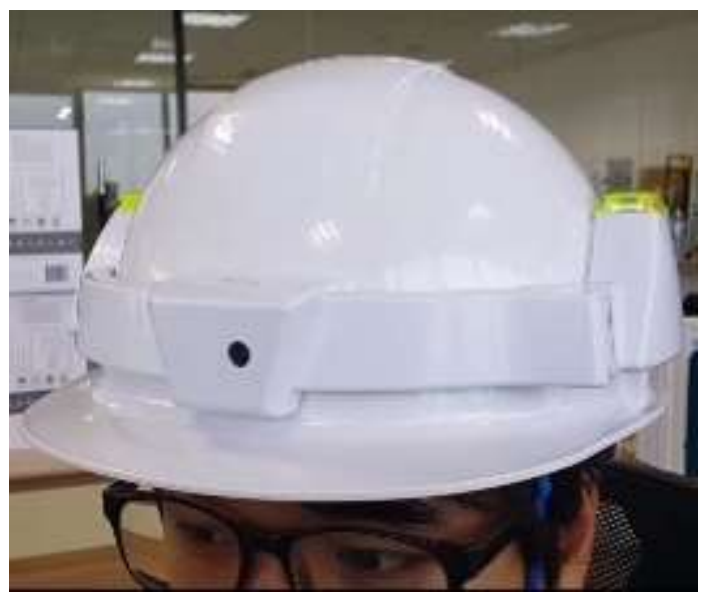

Figure 4.3. Final Implementation of the Smart Helmet

The smart helmet proposed in this study can obtain protective gear safety certification in South Korea and has a replacement cycle of approximately three months for itself and an unlimited replacement cycle for the wireless module. It allows for emergency aid by checking the worker's condition within the golden time when an accident takes place and also potentially minimizes the loss of life on a national level. It would also minimize economic losses due to accidents in light of decreased production costs and shortened production times in industrial settings, and contribute to the advancement of smart technology.

\section{Conclusion}

This study proposes a smart helmet that is entitled to protective gear safety certification in South Korea and has a replacement cycle of approximately three months for itself and an unlimited replacement cycle for the wireless module, and also proposes providing workers with safety related knowledge and techniques through various safety training programs at work sites to reduce accidents in industrial settings. Using the smart helmet would allow for emergency aid by checking the worker's condition within the golden time when an accident takes place and would also minimize the loss of life on a national level. It would also minimize economic losses due to accidents in light of decreased production costs and shortened production times in industrial settings, and contribute to the advancement of smart technology. It is also possible to develop a smart safety control system through additional studies.

\section{References}

[1] K.H. Chung, "Fatal accident analysis and safety management improvement in the construction industry", The Korea Institute of Building Construction, vol. 15, no. 1, (2015), pp. 11-18.

[2] Y.S. Kim and J.K. Park, "A Field Survey on the Development of Safety Management Information System in Construction Management", Journal of The Korean Society of Safety, vol. 19, no. 2, (2004), pp. 75-87.

[3] S.S. Go and H. Song, "Development of the Construction Safety Information System", Journal of The Korean Society of Safety, vol. 16, no. 4, (2001), pp. 140-146.

[4] H.S. Ahn, J.Y. Lee, M.H. Jang, S.J. Choi and S.S. Go, "Development of Safety Management Software Related to the Safety Level of Small \& Medium-Sized Construction Sites", Journal of The Korean Society of Safety, vol .14, no. 2, (1999), pp. 170-177.

[5] B.H. Ryu, H.S. Kim, S.G. Jeong, W.J. Jung and S.W. Lee, "Analysis of the causes of fall accidents and preventive measures in the manufacturing industry - Focus on the use of portable ladders - Center for Safety \& Hygiene Research”, Occupational Safety and Health Research Institute, (2008). 
[6] J.H. Jung, "Survey of the Status of the Occupational Safety and Health Market and Industry for Their Systematic Development", Occupational Safety and Health Research Institute, (2009).

[7] J.H. Kim, S.H. Seo, W.Ch. Shin, J.S. Kim and S.J. Choi, "Survey and Study on Personal Protective Equipment and Protective Devices", Occupational Safety and Health Research Institute, (2011).

[8] D.H. Choi and J.W. Choi, "Survey of Fall Accidents and Study of Safety Models in Roof Work", Occupational Safety and Health Research Institute, (2011).

[9] S.W. Choi, Research on the Roadmap Building between IT and Safety Technology, Occupational Safety and Health Research Institute, (2014).

[10] I.Y. Song, "A Study on the special analysis of the Fall 2.5 of injuries and the development of safety devices", Occupational Safety and Health Research Institute, (2007).

[11] M.S. Jang, M.J. Yeom, I.Y. Song, O.S. Kwon and E.H. Lee, "Check System for wearing hardhats with a wireless sensor network”, 2007 CICS, vol. 1. no. 1, (2007), pp. 209-210.

[12] S.Y. Hong, S.H. Lee, B.M. Kim, M.S. Jang and E.H. Lee, "Implementation of a hardhat for safety at construction sites", The Institute of Electronics Engineers of Korea, vol. 36, no. 1, (2013), pp. 166391642. 